\title{
A new section and two new species of Mycena
}

\section{Aravindakshan DM and Manimohan P}

Department of Botany, University of Calicut, Kerala, 673 635, India

Aravindakshan DM, Manimohan P 2013 - A new section and two new species of Mycena. Mycosphere 4(5), 930-935, Doi 10.5943/mycosphere/4/5/5

\begin{abstract}
A new section, sect. Spinosae, is proposed for species of the agaric genus Mycena that have both thin-walled pileocystidia and diverticulate hyphae on the pileipellis. Two new species, Mycena mridula and M. rasada, are proposed in the new section and five other species of Mycena, currently assigned to other sections, are considered as belonging to it. A key is provided for all species included in the proposed section.
\end{abstract}

Key words - Agaricales - Basidiomycota - biodiversity - Mycenaceae - taxonomy

\section{Introduction}

There are several species of Mycena (Mycenaceae, Agaricales, Basidiomycota) that possess hairs or spines over the pileus. Mycena sect. Longisetae is characterized by the presence of erect, thick-walled pileosetae at least in the primordial stage. About six species of Mycena sect. Basipedes (M. stylobates (Pers.) P. Kumm., M. tenuispinosa J. Favre, M. spinosa Métrod, M. quadratipes Métrod, M. pseudoseta Desjardin, Boonprat. \& Hywel-Jones, M. mimicoseta Desjardin, Boonprat. \& Hywel-Jones) also have pileus spines, but in those species, the spines are formed of bundles of pileipellis hyphae. Apart from these two groups of spinose species of Mycena, there are a few spinose species, currently scattered in several sections that have both hyphae of the pileipellis with excrescences, and thin-walled pileocystidia. These include Mycena setigera Métrod, M. setulipes Métrod, M. paediscula Berk. \& Broome, M. dermatogloea Desjardin, Boonprat. \& Hywel-Jones and Mycena variicystis Boonprat. The first two species were placed in sect. Ciliatae subsect. Setosae by Métrod (1949). Desjardin et al. (2003) placed M. paediscula and M. dermatogloea in a redefined sect. Polyadelphia. Mycena variicystis, reported from Thailand was placed tentatively in the sect. Longisetae by Boonpratuang (2009), although thin-walled pileocystidia is an unusual feature in that section.

In the course of our recent studies on the genus Mycena in Kerala state, we came across two new species that also have both hyphae of the pileipellis with excrescences and thin-walled pileocystidia. These two species are described here as new and a new section is proposed to accommodate these species as well as the five species mentioned above as they form a coherent group distinct from all sections hitherto recognized in Mycena.

\section{Materials \& Methods}

Conventional morphology-based taxonomic methods were employed for this study. Microscopic observations were made on material stained with $1 \%$ aqueous solution of Congo red and mounted in 3\% aqueous $\mathrm{KOH}$. Melzer's reagent was used to observe whether the spores and 
tissues were amyloid. For evaluation of the range of spore-size, 20 basidiospores each from one specimen of each collection cited were measured. Colour codes used in the descriptions are from Kornerup \& Wanscher (1978). Except one, all the examined collections cited, including the holotypes, are deposited at the Kew (Mycology) herbarium and their Kew accession numbers (e.g., $\mathrm{K}(\mathrm{M})$ 180393) are provided.

\section{Results and Discussion}

\section{Taxonomy}

Mycena section Spinosae Aravind. \& Manim., sect. nov.

\section{MycoBank MB 804511}

Pileipellis composed of both diverticulate hyphae and thin-walled pileocystidia.

Basidiomata small to medium-sized. Pileus dry or viscid, sometimes with a separable pellicle, covered with fine pubescence, cream white, yellow, olivaceous, grey or greyish brown, translucent-striate. Lamellae adnate, or with a decurrent tooth, rarely psuedocollariate, arcuate, or vein-like, white, grey or yellowish with paler or darker edge. Stipe hollow, fragile or filiform, pruinose or pubescent, rarely glabrous, base with fine radiating fibrils, rarely insititious or rooting. Basidiospores ellipsoid, amyloid or inamyloid. Basidia 2- or 4-spored. Cheilocystidia clavate, cylindrical or fusoid, smooth or with excrescences. Pleurocystidia absent. Hyphae of the pileipellis diverticulate. Pileocystidia originating either from the pileipellis or from the pileus trama, thinwalled, hyaline, smooth. Hyphae of the stipitipellis smooth, rarely with excrescences. Caulocystidia absent or similar to pileocystidia if present.

Type species - Mycena mridula Aravind. \& Manim.

The following key differentiates all Mycena species considered as belonging to sect. Spinosae:

1. Pileocystidia originating from hyphae of the pileipellis ...................................... 2

1. Pileocystidia originating from hyphae of the pileus trama …............................................... 3

2. Stipe with a discoid base; hyphae of the pileipellis terminating in acanthocysts; basidiospores ellipsoid; caulocystidia absent Mycena variicystis

2. Stipe without a discoid base; hyphae of the pileipellis not terminating in acanthocysts; basidiospores oblong-ellipsoid to subcylindrical; caulocystidia present.................. Mycena mridula

3. Hyphae of the pileipellis with acanthocystoid terminal cells; pileogloeocystidia present ......... 4

3. Hyphae of the pileipellis without acanthocystoid terminal cells; pileogloeocystidia absent .... 5

4. Basidiomata growing on senescent leaves; stipe base orange; basidiospores 7-9 $\mu \mathrm{m}$ long; cheilocystidia absent Mycena dermatogloea

4. Basidiomata growing on dead wood; Stipe base pale brown; basidiospores 5.5-7.5 $\mu \mathrm{m}$ long; cheilogloeocystidia present, lageniform .... Mycena paediscula

5. Basidiomata growing on humus; stipe base rooting ....................................... Mycena setulipes

5. Basidiomata growing on wood, twigs or bark of trees; stipe base not rooting ................... 6

6. Cheilocystidia exuding a yellowish resinous substance; caulocystidia present ...... Mycena rasada

6. Cheilocystidia without any exudation; caulocystidia absent Mycena setigera

Notes - Mycena globuliformis Segedin (1991), a New Zealand species presently assigned to sect. Longisetae, is also reported to have "large bundles of thin walled hairs" as well as diverticulate hyphae on the pileal surface and therefore can be considered as belonging sect. Spinosae. However, that species is reported to have a very complex pileipellis structure with highly distinctive suprapellis, pellis and subpellis layers. Additionally, it has thick-walled caulocystidia. Also, Segedin (1991) has neither described nor illustrated the morphology of the pileal hairs. Owing to these reasons, we refrain from considering Mycena globuliformis in sect. Spinosae. 
Characterized by very small, whitish basidiomata; greyish pileus dotted with spinules; oblong-ellipsoid to subcylindrical, amyloid basidiospores; thin-walled, conic or fusoid pileocystidia with an acuminate apex and a base ensheathed with pileipellis hyphae; and smooth stipitipellis hyphae with caulocystidia. Differing from Mycena variicytis in having basidiomata that grow on decaying leaves, stipe without a discoid base, hyphae of the pileipellis not terminating in acanthocysts, much smaller basidiospores, and caulocystidia.

Etymology - mridula (Sanskrit), tender.

Basidiomata very small, delicate. Pileus $2.5-4.5 \mathrm{~mm}$ diam., conic when young, becoming convex with age; surface greyish at the centre and on the striations, whitish elsewhere, translucentstriate, with scattered spinules beset with a central hair; margin straight, entire when young, becoming fissile with age. Lamellae up to 15 reaching the stipe, adnate, seceding, pale greyish, less than $0.5 \mathrm{~mm}$ thick, subdistant, with lamellulae of one length; edge finely torn, white, paler than the sides. Stipe 30-40 $\times 0.5 \mathrm{~mm}$, central, terete, almost equal or slightly tapering towards apex, hollow; surface translucent, whitish to greyish, finely pubescent; base lacking both basal disc and basal mycelium. Context not conspicuous. Odour not distinctive. Taste not recorded.

Basidiospores $(6-) 7-9(-10) \times 3-4.5(8.275 \pm 0.769 \times 4.025 \pm 0.412) \mu \mathrm{m}, \mathrm{Q}=1.77-2.33$, $\mathrm{Qm}=2.02$, oblong-ellipsoid to subcylindrical, thin-walled, hyaline, smooth, strongly amyloid, with a few guttules. Basidia 11-13 $\times 6.5-7 \mu \mathrm{m}$, obovoid, bearing 4 sterigmata up to $3 \mu \mathrm{m}$ long. Cheilocystidia 9.5-27.5 $\times 6-9.5 \mu \mathrm{m}$, clavate to cylindrical, thin-walled, hyaline, with cylindrical excrescences $(1-3 \times 1-1.5 \mu \mathrm{m})$. Pleurocystidia none. Lamellar trama regular to subregular; hyphae 2.5-17 $\mu \mathrm{m}$ wide, thin-walled, hyaline, very faintly vinoid in Melzer's reagent. Subhymenium pseudoparenchymatous. Pileus trama subregular; hyphae 13-24 $\mu \mathrm{m}$ wide, thin-walled, hyaline, very faintly vinoid in Melzer's reagent. Pileipellis a cutis; hyphae 1-3 $\mu \mathrm{m}$ wide, thin-walled, hyaline, with excrescences $(1-5 \times 0.5-1 \mu \mathrm{m})$; pileocystidia $33-125 \times 7-10 \mu \mathrm{m}$, narrowly conic or fusoid with an acuminate apex, thin-walled, densely covered with the narrow pileipellis hyphae towards the base and thus forming the spinules on the pileal surface. Stipitipellis a cutis of smooth hyphae with intercalary or terminal caulocystidia; hyphae $1.5-4.5 \mu \mathrm{m}$ wide, thin-walled, hyaline. Caulocystidia 14-111.5 $\times 4.5-7 \mu \mathrm{m}$, narrowly conic with acuminate apex, often with apical or basal protrusions $(8-28 \times 1-3 \mu \mathrm{m})$, often branching, thin-walled. Stipe trama pale greyish in water, vinoid in Melzer's reagent. Clamp connections seen on all hyphae.

On decaying leaves, scattered or in groups, June.

Distribution - known only from the type locality.

Material examined - India, Kerala State, Kannur District, Kalarivathukkal: 17 June 2009, D.M. Aravindakshan DM268 (K(M) 180393, holotype).

Notes - Very small, delicate, whitish basidiomata; greyish pileus dotted with fine spinules; oblong-ellipsoid to subcylindrical and amyloid basidiospores; thin-walled, conic or fusoid pileocystidia with acuminate apex and ensheathed at the base with pileipellis hyphae; and smooth stipitipellis hyphae with lateral or terminal caulocystidia are the diagnostic features of the present species.

As already noted, Mycena setigera, M. setulipes, $M$. paediscula, M. dermatogloea, $M$. variicystis and M. globuliformis are the Mycena species thus far reported having both hyphae of the pileipellis with diverticula or excrescences, and thin-walled pileocystidia. In all the first four species listed above, the pileocystidia are subcuticular in origin. In addition to that differing character, M. setigera differs from the present species in having long (up to $35 \mu \mathrm{m}$ ), narrow, cylindrical, smooth cheilocystidia. Mycena setulipes is a rooting species with small pileocystidia and very narrow (up to $1.5 \mu \mathrm{m}$ ), cylindrical, subcapitate, smooth, cheilocystidia. Mycena variicystis is somewhat similar to the present species in having cheilocystidia with excrescences, similar type of pileocystidia, non-gelatinous hyphae of the pileipellis and in lacking pleurocystidia. But that 


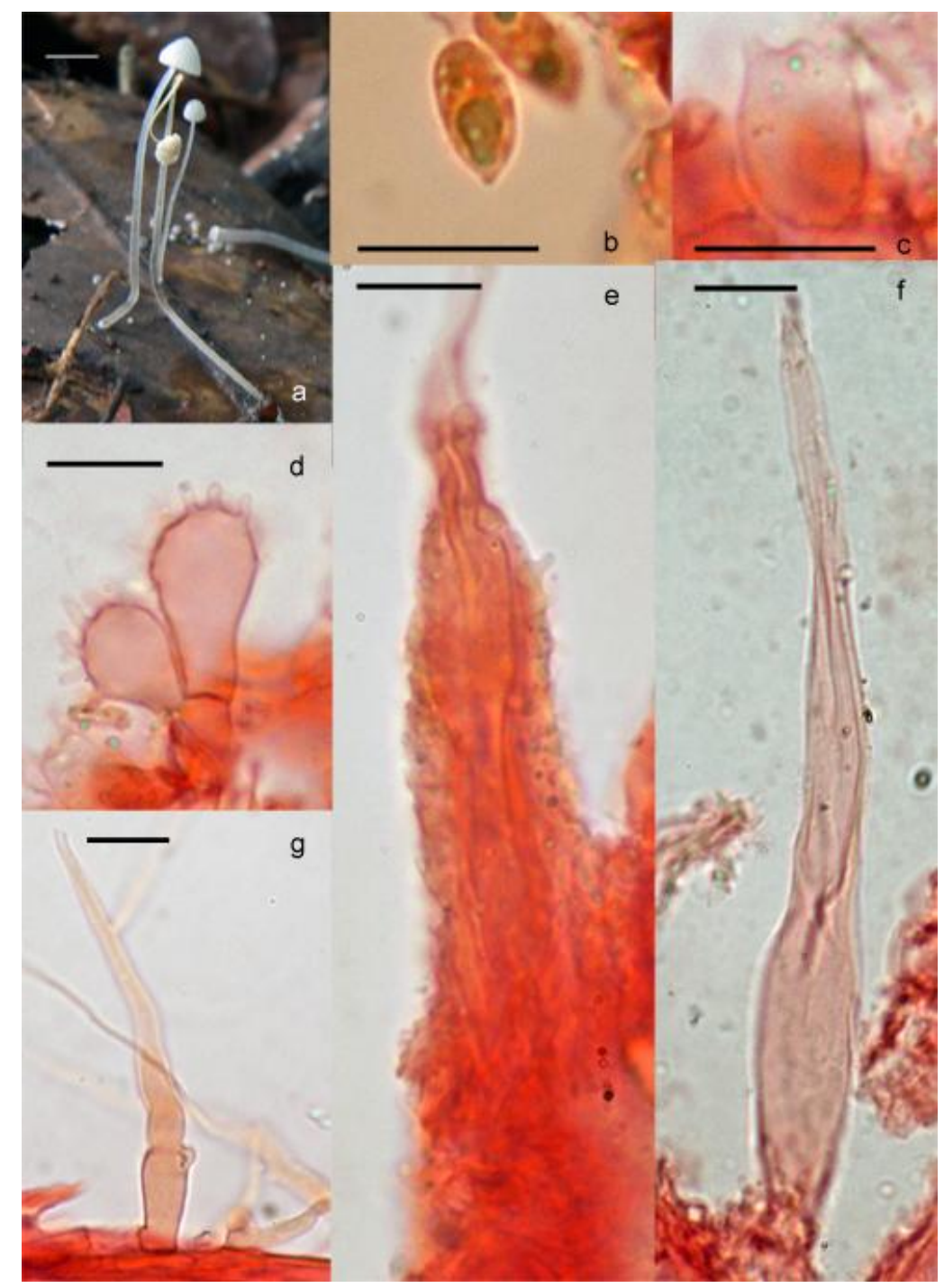

Fig. 1 a-g - Mycena mridula. a Basidiomata. b Basidiospore. c Basidium. d Cheilocystidia. e Spinule on the pileus. f Pileocystidium. g Caulocystidium. - Bars $=1 \mathrm{~mm}$ for basidiomata and 10 $\mu \mathrm{m}$ for micro-structures.

species differs in having smaller stipe (up to $15 \mathrm{~mm}$ long), pale brown pileus, much larger ( $8-15 \times$ 4-7 $\mu \mathrm{m}$ ) basidiospores, larger cheilocystidia with much larger apical appendages (up to $12 \mu \mathrm{m}$ long), distinct acanthocysts, strongly dextrinoid pileus trama and in lacking caulocystidia. Mycena globuliformis differs in having a complex pileipellis structure and thick-walled caulocystidia.

Mycena rasada Aravind. \& Manim., sp. nov.

Fig. $2 \mathrm{a}-\mathrm{h}$

MycoBank MB 804510

Holotype - K(M)180098

Characterized by small basidiomata densely covered with fine, thin-walled hairs; slightly gelatinized hyphae of the pileipellis forming a separable pellicle; oblong-ellipsoid to subamygdaliform, inamyloid basidiospores; cylindrical, exudative cheilocystidia with capitate apex; and long, thin-walled obclavate pileocystidia and caulocystidia. Differing from Mycena setigera in having non-exudative cheilocysidia and in lacking caulocystidia.

Etymology - rasada (Sanskrit), exuding resin.

Basidiomata small. Pileus 1.5-4.5 mm diam., 2-3 mm high, parabolic or hemispheric when young, becoming convex with age; surface dark brown (6F6) at the centre and on the striations, and greyish brown $(6 \mathrm{E} 4,6 \mathrm{D} 4,5 \mathrm{E} 4)$ elsewhere, translucent-striate, finely pubescent all over, with a 
separable pellicle; margin straight, finely hairy when young, becoming slightly undulate with age. Lamellae 8-13 reaching the stipe, narrowly adnate or adnate with a small decurrent tooth, whitish, $0.5 \mathrm{~mm}$ wide, subdistant, with lamellulae of 1-2 lengths; edge entire, concolorous with the sides, often with yellow resinous exudation. Stipe $7-45 \times 0.25-0.5 \mathrm{~mm}$, central, terete, slightly tapering towards apex, hollow; surface translucent, white, pubescent all over; base with strigose basal mycelium. Context not conspicuous, less than $0.5 \mathrm{~mm}$ wide, concolorous with the pileus. Odour and taste not recorded.

Basidiospores $(6.5-) 7.5-10(-11) \times(2.5-) 3-4.5(9.213 \pm 0.802 \times 3.594 \pm 0.606) \mu \mathrm{m}, \mathrm{Q}=$ 1.78-3.5, Qm = 2.63, oblong-ellipsoid to subamygdaliform, thin-walled, hyaline, smooth, inamyloid. Basidia 13.5-19(-21.5) $\times 7-8.5(-13) \mu \mathrm{m}$, subcylindric to clavate, bearing 4 sterigmata up to $5 \mu \mathrm{m}$ long. Lamella-edge sterile. Cheilocystidia (12-)14-38 $\times 3-6 \mu \mathrm{m}$, cylindrical to narrowly clavate, often with a subcapitate apex, thin-walled, exuding a yellowish, resinous substance in which spores get attached. Pleurocystidia none. Lamellar trama subregular; hyphae 2$28 \mu \mathrm{m}$ wide, thin-walled, hyaline, inamyloid in Melzer's reagent. Subhymenium ramose. Pileus trama interwoven; hyphae $10-27(-35) \mu \mathrm{m}$ wide, thin- to slightly $(0.5 \mu \mathrm{m})$ thick-walled, pale greyish or brownish, very faintly vinoid in Melzer's reagent. Pileipellis a cutis, narrow; hyphae 1.5-4 $\mu \mathrm{m}$ wide, thin-walled, hyaline, slightly gelatinized, with simple or branched, short, cylindrical excrescences $(1-4 \times 0.5-2 \mu \mathrm{m})$; pileocystidia 40-206 $\times 10-25 \mu \mathrm{m}$, obclavate with a long, flexuous, gradually tapering apex, originate from the pileus trama, thin-walled, hyaline. Pileus marginal cells 38-160 × 12-17 $\mu \mathrm{m}$, fusoid to obclavate, often with a tapering apex or a small beak, sometimes similar to pileocystidia, thin-walled, hyaline. Stipitipellis a cutis; hyphae 1.5-7.5 $\mu \mathrm{m}$ wide, thin-walled, hyaline or pale yellowish, smooth. Caulocystidia (18-)48-126 × (5.5-)10-19 $\mu \mathrm{m}$, similar to pileocystidia, thin-walled, hyaline. Stipe trama very faintly vinoid in Melzer's reagent. Clamp connections seen on all hyphae.

On decaying twigs, solitary or scattered, June-November.

Distribution - known only from three different localities in Kerala State, India.

Material examined - India, Kerala State, Malappuram District, Calicut University Campus: 10 June 2009, D.M. Aravindakshan DM245; Thrissur District, Peechi: 7 July 2010, D.M. Aravindakshan DM447 (K(M)180098, holotype); Palakkad District, Pattambi, Rayiranallur: 2 November 2010, D.M. Aravindakshan DM514 (K(M) 180380).

Notes - Small basidiomata densely covered with fine, thin-walled hairs; slightly gelatinized hyphae of the pileipellis forming a separable pellicle; oblong-ellipsoid to subamygdaliform, inamyloid basidiospores; cylindrical, exudative cheilocystidia with capitate apex; and long, thinwalled obclavate pileocystidia and caulocystidia are the diagnostic features of the present species. The combined presence of both diverticulate hyphae and thin-walled pileocystidia in the pileipellis warrants placement of this species under sect. Spinosae.

Mycena setigera shares several similar features with the present species but differs in having non-exudative cheilocysidia and in lacking caulocystidia. Mycena globuliformis is comparable with the present species in having similar-sized basidiospores, smooth cheilocystidia, and gelatinized hyphae in the pileipellis. But characters such as the stipe basal disc lined with a black margin, strongly amyloid basidiospores, much longer, non-exudative cheilocystidia, complex pileipellis with subpellis composed of thick-walled elements, and thick-walled caulocystidia make M. globuliformis a different species. Mycena dermatogloea has similar-sized basidiospores and lacks pleurocystidia. Additionally, its pileocystidia is exudative like the cheilocystidia of the present species. But that species differs in having, smaller basidiomata with orange or yellowish white pileus, orange stipe base, amyloid basidiospores, exudative pileo- and caulocystidia, pileogloeocystidia that originate from the gleoplerous hyphae of pileus trama, and diverticulate hyphae of the stipitipellis and in lacking cheilocystidia. Mycena paediscula has exudative cheilocystidia and habitat on the decaying wood just like the present species, but it differs in having smaller basidiospores, lageniform cheilogloeocystidia, and pileogloeocystidia. 


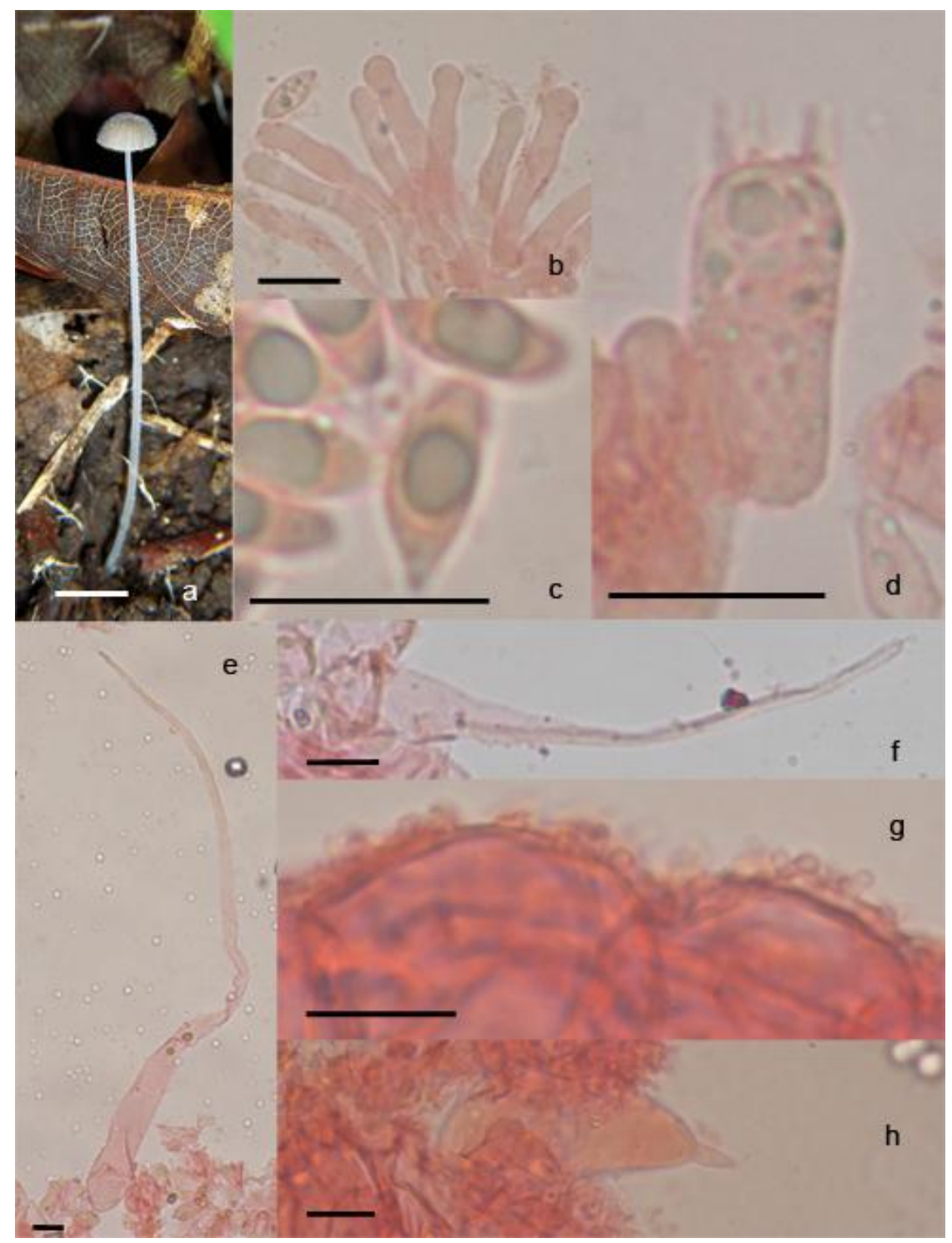

Fig. 2 a-h - Mycena rasada. a Basidioma. b Cheilocystidia. c Basidiospore. d Basidium. e Pileocystidium. f Caulocystidium. g Hyphae of the pileipellis. h Pileus marginal cell. - Bars $=5$ $\mathrm{mm}$ for basidioma and $10 \mu \mathrm{m}$ for micro-structures.

\section{Acknowledgements}

One of us (DMA) acknowledges financial assistance from the University of Calicut in the form of a PhD fellowship and is thankful to Mr K. N. Anil Raj for his help in collecting mycenas.

\section{References}

Boonpratuang T. 2009 - Mycena variicystis, a new spinose species from Phru Toh Daeng Peat Swamp. Mycotaxon 109, 185-188.

Desjardin DE, Boonpratuang T, Hywel-Jones N. 2003 - New spinose species of Mycena in sections Basipedes and Polyadelphia from Thailand. Fungal Diversity 12, 7-17.

Kornerup A, Wanscher JH. 1978 - Methuen Handbook of Colour. 3rd Ed. Eyre Methuen, London.

Métrod G. 1949 - Les Mycènes de Madagascar (Mycena, Corrugaria, Pterospora). Laboratoire de Cryptogamie du Museum National d'Histoire Naturelle, Paris.

Segedin BP. 1991 - Studies in the Agaricales of New Zealand: some Mycena species in sections Longisetae, Polyadelpha, Rubromarginatae, Galactopoda, Lactipedes, and Calodontes. New Zealand Journal of Botany 29, 43-62. 